\title{
Efficient algorithms for computing the largest eigenvalue of a nonnegative tensor
}

\author{
Guanglu ZHOU ${ }^{1}$, Liqun $\mathbf{Q I}^{2}$, Soon-Yi WU ${ }^{3}$ \\ 1 Department of Mathematics and Statistics, Curtin University, Perth, Australia \\ 2 Department of Applied Mathematics, The Hong Kong Polytechnic University, Hong Kong, \\ China \\ 3 Institute of Applied Mathematics, Cheng-Kung University, Tainan, China
}

(c) Higher Education Press and Springer-Verlag Berlin Heidelberg 2012

\begin{abstract}
Consider the problem of computing the largest eigenvalue for nonnegative tensors. In this paper, we establish the Q-linear convergence of a power type algorithm for this problem under a weak irreducibility condition. Moreover, we present a convergent algorithm for calculating the largest eigenvalue for any nonnegative tensors.
\end{abstract}

Keywords Eigenvalue, nonnegative tensor, power method, linear convergence MSC 65F15, 65F10, 15A18, 15A69

\section{Introduction}

Let $\mathbb{R}$ be the real field. In this paper, we consider an $m$-order $n$-dimensional tensor $\mathscr{A}$ consisting of $n^{m}$ entries in $\mathbb{R}$ :

$$
\mathscr{A}=\left(a_{i_{1} i_{2} \cdots i_{m}}\right), \quad a_{i_{1} i_{2} \cdots i_{m}} \in \mathbb{R}, \quad 1 \leqslant i_{1}, i_{2}, \ldots, i_{m} \leqslant n .
$$

$\mathscr{A}$ is called nonnegative (resp. positive) if $a_{i_{1} i_{2} \cdots i_{m}} \geqslant 0$ (resp. $a_{i_{1} i_{2} \cdots i_{m}}>0$ ). When $m=2, \mathscr{A}$ is a matrix. When $m \geqslant 3, \mathscr{A}$ is called a higher-order tensor. Tensors play an important role in physics, engineering, and mathematics. Applications of tensors include data analysis and mining, information science, signal and image processing, and computational biology, etc. See [24] and references therein.

To an $n$-dimensional column vector $x=\left[x_{1}, x_{2}, \ldots, x_{n}\right]^{\mathrm{T}} \in \mathbb{R}^{n}$, real or complex, we define an $n$-dimensional column vector:

$$
\mathscr{A} x^{m-1}:=\left(\sum_{i_{2}, \ldots, i_{m}=1}^{n} a_{i i_{2} \cdots i_{m}} x_{i_{2}} \cdots x_{i_{m}}\right)_{1 \leqslant i \leqslant n} .
$$

Received March 19, 2012; accepted October 11, 2012

Corresponding author: Guanglu ZHOU, E-mail: G.Zhou@curtin.edu.au 
Definition 1.1 Let $\mathscr{A}$ be an $m$-order $n$-dimensional tensor and $\mathbb{C}$ be the set of all complex numbers. Assume that $\mathscr{A} x^{m-1}$ is not identical to zero. We say $(\lambda, x) \in \mathbb{C} \times\left(\mathbb{C}^{n} \backslash\{0\}\right)$ is an eigenvalue-eigenvector of $\mathscr{A}$ if

$$
\mathscr{A} x^{m-1}=\lambda x^{[m-1]} .
$$

Here,

$$
x^{[\alpha]}=\left[x_{1}^{\alpha}, x_{2}^{\alpha}, \ldots, x_{n}^{\alpha}\right]^{\mathrm{T}} .
$$

This definition was introduced in $[3,16,23]$. Unlike matrices, eigenvalue problems for higher-order tensors are nonlinear. Applications of eigenvalues of higher-order tensors include medical resonance imaging [1,26], higher-order Markov chains [20], positive definiteness of even-order multivariate forms in automatical control [21], and best-rank one approximation in data analysis $[8,14,15,25,27]$, etc.

Recently, eigenvalue problems for higher-order tensors have gained special attention in the realm of numerical multilinear algebra. In particular, the Perron-Frobenius theorem for eigenvalues of nonnegative tensors have been established in $[3,4,6]$. Friedland et al. [10] established the Perron-Frobenius theorem for homogeneous monotone maps. The Perron-Frobenius Theorem for nonnegative tensors is related to measuring higher order connectivity in linked objects [17] and hyper-graphs [2,9]. Subsequently, based on the minimax theorem for nonnegative tensors in [3], $\mathrm{Ng}$ et al. [20] proposed a power type method for computing the largest eigenvalue and the corresponding eigenvector of an irreducible nonnegative tensor. This method is an extension of a method of Collatz $[7,28,29]$ for calculating the spectral radius of an irreducible nonnegative matrix. In [5], the convergence of this method for primitive nonnegative tensors has been established. For the definition of a primitive nonnegative tensor, we will give it in the next section. A primitive nonnegative tensor $\mathscr{A}$ is irreducible, but the converse is false; see [5]. In [18,32], the authors proposed an updated version of the Ng-Qi-Zhou method [20] and it has been proved that this algorithm is always convergent for any irreducible nonnegative tensors. Furthermore, Zhang and Qi [31], Zhang et al. [32] established the linear convergence of the Ng-Qi-Zhou method and its updated version for essentially positive tensors and weakly positive tensors, respectively. A weakly positive tensor is irreducible, but the converse is false; see [32].

In this paper, we establish, in Section 3, the Q-linear convergence of power algorithm [18,32] under the weak irreducibility condition; see Theorem 3.2 for details. In addition, based on [30, Theorem 2.3], we present a convergent algorithm for calculating the largest eigenvalue and a corresponding nonnegative eigenvector for any nonnegative tensors. Recently, A power algorithm for polynomial eigenvalue problems has been introduced in [10] and the R-linear convergence of the power algorithm has been established under the weak primitivity condition. Furthermore, global linear convergence of this algorithm has been studied in [13]. 
Throughout this paper, we use $\mathbb{R}^{n}$ (resp. $\mathbb{C}^{n}$ ) to denote the $n$-dimensional real (resp. complex) space. Let

$$
P_{n}=\left\{x \in \mathbb{R}^{n}: x_{i} \geqslant 0,1 \leqslant i \leqslant n\right\}
$$

and

$$
\operatorname{int}\left(P_{n}\right)=\left\{x \in \mathbb{R}^{n}: x_{i}>0,1 \leqslant i \leqslant n\right\} .
$$

Matrices are denoted by italic capitals $(A, B, \ldots)$ and higher-order tensors are written as calligraphic capitals $(\mathscr{A}, \mathscr{B}, \ldots)$.

\section{Preliminaries}

In this section, we will give some definitions and results about nonnegative matrices and nonnegative tensors, which will be used in the next section.

\subsection{Nonnegative matrices}

Let $M$ be an $n \times n$ nonnegative matrix. The graph associated to $M$ (see [28, Chap. 2]), $\mathscr{G}(M)$, is the directed graph with vertices $1,2, \ldots, n$ and an edge from $i$ to $j$ if and only if $M_{i j} \neq 0$. A directed graph is said to be strongly connected if there is a directed path between any two distinct vertices. The matrix $M$ is called irreducible if the graph $\mathscr{G}(M)$ is strongly connected. We say that $M$ is primitive if the graph $\mathscr{G}(M)$ is strongly connected and the greatest common divisor (gcd) of the lengths of its circuits is equal to one. An irreducible matrix with a nonzero main diagonal is primitive ([19, Corollary 3.2]). Let $\sigma(M)$ denote the spectrum of $M$, the set of all eigenvalues of $M$. The spectral radius of $M$, denoted by $\rho(M)$, is the maximum distance of an eigenvalue from the origin, i.e.,

$$
\rho(M)=\max \{|\lambda|: \lambda \in \sigma(M)\} .
$$

The classical Perron-Frobenius theorem for nonnegative matrices may be stated as follows (see [28, Chap. 2]).

Theorem 2.1 If $M$ is an irreducible nonnegative matrix, then $M$ has an eigenvector $u \in \operatorname{int}\left(P_{n}\right)$, unique up to a scale multiple, whose associated eigenvalue is the spectral radius of $M, \rho(M)$. Moreover, $\rho(M)$ is a simple root of the characteristic equation of $M$. Furthermore, if the nonnegative matrix $M$ is primitive, then

$$
\rho(M)>|\lambda|, \quad \forall \lambda \in \sigma(M) \backslash\{\rho(M)\} .
$$

If $A$ is an $n \times n$ matrix, then the spectral norm [28] is defined as

$$
\|A\|=\sup _{x \neq 0} \frac{\|A x\|}{\|x\|} .
$$

Here, $\|\cdot\|$ denotes a vector norm on the vector space $\mathbb{R}^{n}$. The relationships between the spectral radius of $A$ and the spectral norm of $A$ are as follows. 
Proposition $2.1[11,12]$ The spectral radius of an $n \times n$ matrix $A$ is characterized by the equality

$$
\rho(A)=\inf _{\|\cdot\| \in \mathscr{N}}\|A\|,
$$

where $\mathscr{N}$ denotes the set of all possible spectral norms of $A$. For any $\varepsilon>0$, there exists a spectral norm $\|\cdot\|_{\varepsilon} \in \mathscr{N}$ such that

$$
\|A\|_{\varepsilon} \leqslant \rho(A)+\varepsilon .
$$

\subsection{Nonnegative tensors}

Let $\mathscr{A}$ be an $m$-order $n$-dimensional nonnegative tensor. The spectral radius of $\mathscr{A}$ is defined as

$$
\rho(\mathscr{A})=\max \{|\lambda|: \lambda \text { is an eigenvalue of } \mathscr{A}\} .
$$

Definition 2.2 [3] An $m$-order $n$-dimensional tensor $\mathscr{A}$ is called reducible if there exists a nonempty proper index subset $I \subset\{1,2, \ldots, n\}$ such that

$$
a_{i_{1} i_{2} \cdots i_{m}}=0, \quad \forall i_{1} \in I, \forall i_{2}, \ldots, i_{m} \notin I .
$$

If $\mathscr{A}$ is not reducible, then we call $\mathscr{A}$ irreducible.

Let $\mathscr{A}$ be an $m$-order $n$-dimensional nonnegative tensor. The graph associated to $\mathscr{A}, \mathscr{G}(\mathscr{A})$, is the directed graph with vertices $1,2, \ldots, n$ and an edge from $i$ to $j$ if and only if $a_{i i_{2} \cdots i_{m}} \neq 0$ for some $i_{l}=j, l=2,3, \ldots, m$.

Definition 2.3 [10] An $m$-order $n$-dimensional tensor $\mathscr{A}$ is called weakly irreducible if $\mathscr{G}(\mathscr{A})$ is strongly connected. If $\mathscr{G}(\mathscr{A})$ is strongly connected and the greatest common divisor (gcd) of the lengths of its circuits is equal to one, then $\mathscr{A}$ is called weakly primitive.

We have the following proposition.

Proposition $2.2[10] \quad$ If nonnegative tensor $\mathscr{A}$ is irreducible, then $\mathscr{A}$ is weakly irreducible. For $m=2, \mathscr{A}$ is irreducible if and only if $\mathscr{A}$ is weakly irreducible.

Let $\mathscr{I}$ be the $m$-order $n$-dimensional unit tensor whose entries are

$$
I_{i_{1} i_{2} \cdots i_{m}}= \begin{cases}1, & i_{1}=i_{2}=\cdots=i_{m} \\ 0, & \text { otherwise }\end{cases}
$$

Proposition 2.3 [13] If nonnegative tensor $\mathscr{A}$ is weakly irreducible, then $\mathscr{A}+$ $\mathscr{I}$ is weakly primitive.

In [3], the Perron-Frobenius theorem and the well-known Collatz [7] minimax theorem for irreducible nonnegative matrices have been extended to irreducible nonnegative tensors. In the following, we state these results for reference. 
Theorem 2.2 [3] If $\mathscr{A}$ is an irreducible nonnegative tensor of order $m$ and dimension $n$, then there exist $\lambda_{0}>0$ and $x_{0}>0, x_{0} \in R^{n}$ such that

$$
\mathscr{A} x_{0}^{m-1}=\lambda_{0} x_{0}^{[m-1]} \text {. }
$$

Moreover, if $\lambda$ is an eigenvalue with nonnegative eigenvector, then $\lambda=\lambda_{0}$. If $\lambda$ is an eigenvalue of $\mathscr{A}$, then $|\lambda| \leqslant \lambda_{0}$.

Theorem 2.3 [3] Assume that $\mathscr{A}$ is an irreducible nonnegative tensor of order $m$ and dimension $n$. Then

$$
\min _{x \in \operatorname{int}(P)} \max _{x_{i}>0} \frac{\left(\mathscr{A} x^{m-1}\right)_{i}}{x_{i}^{m-1}}=\lambda_{0}=\max _{x \in \operatorname{int}(P)} \min _{x_{i}>0} \frac{\left(\mathscr{A} x^{m-1}\right)_{i}}{x_{i}^{m-1}}
$$

where $\lambda_{0}$ is the unique positive eigenvalue corresponding to the positive eigenvector.

Let $\mathscr{A}$ be a nonnegative tensor. For any vector $x \in P_{n}$, we define the following sequence $\left\{\mathscr{A}^{(k)} x\right\}$ :

$$
\begin{gathered}
\mathscr{A}^{(1)} x=\mathscr{A}(x)^{m-1}, \quad z^{(1)}=\left(\mathscr{A}^{(1)} x\right)^{\left[\frac{1}{m-1}\right]}, \\
\mathscr{A}^{(2)} x=\mathscr{A}\left(z^{(1)}\right)^{m-1}, \quad z^{(2)}=\left(\mathscr{A}^{(2)} x\right)^{\left[\frac{1}{m-1}\right]}, \\
\ldots, \\
\mathscr{A}^{(k)} x=\mathscr{A}\left(z^{(k-1)}\right)^{m-1}, \quad z^{(k)}=\left(\mathscr{A}^{(k)} x\right)^{\left[\frac{1}{m-1}\right]}, \quad k \geqslant 2 .
\end{gathered}
$$

Definition 2.4 [5] A nonnegative tensor $\mathscr{A}$ is primitive if there exists a positive integer $k$ such that $\mathscr{A}^{(k)} x \in \operatorname{int}\left(P_{n}\right)$ for any nonzero $x \in P_{n}$.

Clearly, positive tensors and essentially positive tensors [5,22] are primitive. A primitive nonnegative tensor $\mathscr{A}$ is irreducible, but the converse is false [5]. We have the following result.

Theorem 2.4 [5,18] Suppose that $\mathscr{A}$ is an irreducible nonnegative tensor. Let $\mathscr{B}=\mathscr{A}+\mathscr{I}$. Then, we have

(i) $\mathscr{B}$ is primitive;

(ii) if $\lambda$ is the largest eigenvalue of $\mathscr{B}$ and $u$ is a positive eigenvector of $\mathscr{B}$ associated with $\lambda$, then $\lambda-1$ is the largest eigenvalue of $\mathscr{A}$ and $u$ is a positive eigenvector of $\mathscr{A}$ associated with $\lambda-1$.

\section{A power algorithm}

In this section, we state a power type algorithm for calculating the largest eigenvalue of a nonnegative tensor $\mathscr{A}$ by applying the algorithm proposed in [20] to tensor $\mathscr{B}=\mathscr{A}+\mathscr{I}$, and establish the Q-linear convergence of this power algorithm under a weak irreducibility condition. 
For any nonnegative column vector $x \in \mathbb{R}^{n}$, we define $\phi: P_{n} \rightarrow P_{1}$ by

$$
\phi(x)=\sum_{i=1}^{n} x_{i} .
$$

\section{Algorithm 3.1}

Step 0 Choose $x^{(1)} \in \operatorname{int}\left(P_{n}\right)$. Let $\mathscr{B}=\mathscr{A}+\mathscr{I}$, and set $k:=1$.

Step 1 Compute

$$
\begin{gathered}
y^{(k)}=\mathscr{B}\left(x^{(k)}\right)^{m-1}, \\
\underline{\lambda}_{k}=\min _{x_{i}^{(k)}>0} \frac{\left(y^{(k)}\right)_{i}}{\left(x_{i}^{(k)}\right)^{m-1}}, \\
\bar{\lambda}_{k}=\max _{x_{i}^{(k)}>0} \frac{\left(y^{(k)}\right)_{i}}{\left(x_{i}^{(k)}\right)^{m-1}} .
\end{gathered}
$$

Step 2 If $\bar{\lambda}_{k}=\underline{\lambda}_{k}$, then let $\lambda=\bar{\lambda}_{k}$ and stop. Otherwise, compute

$$
x^{(k+1)}=\frac{\left(y^{(k)}\right)^{\left[\frac{1}{m-1}\right]}}{\phi\left(\left(y^{(k)}\right)^{\left[\frac{1}{m-1}\right]}\right)},
$$

replace $k$ by $k+1$ and go to Step 1 .

Theorem 3.1 Suppose that nonnegative tensor $\mathscr{A}$ is weakly irreducible. Let $\mathscr{B}=\mathscr{A}+\mathscr{I}$ and assume that $\lambda$ is the largest eigenvalue of $\mathscr{B}$. Then, Algorithm 3.1 produces a value of $\lambda$ and a corresponding eigenvector $u$ in a finite number of steps, or generates three convergent sequences $\left\{\underline{\lambda}_{k}\right\},\left\{\bar{\lambda}_{k}\right\}$, and $\left\{x^{(k)}\right\}$ such that

$$
\lim _{k \rightarrow \infty} \underline{\lambda}_{k}=\lim _{k \rightarrow \infty} \bar{\lambda}_{k}=\lambda, \quad \lim _{k \rightarrow \infty} x^{(k)}=u .
$$

Furthermore, $\lambda-1$ is the largest eigenvalue of $\mathscr{A}$ associated with the eigenvector $u$.

Proof By Proposition 2.3, $\mathscr{B}$ is weakly primitive. Hence, from [10, Corollary 5.1] and the result (ii) of Theorem 2.4, this theorem holds.

Let

$$
F(x)=\mathscr{B} x^{m-1}, \quad G(x)=F(x)^{\left[\frac{1}{m-1}\right]}, \quad H(x)=\frac{G(x)}{\phi(G(x))} .
$$

Clearly, the sequence $\left\{x^{(k)}\right\}$ in Theorem 3.1 is generated by

$$
x^{(k+1)}=H\left(x^{(k)}\right), \quad k=1,2, \ldots,
$$

and $\phi\left(x^{(k)}\right)=1$ for all $k=1,2, \ldots$ 
Theorem 3.2 Let $\mathscr{A}, \mathscr{B}$, and $\left\{x^{(k)}\right\}$ be as in Theorem 3.1. Then, the convergence rate of the sequence $\left\{x^{(k)}\right\}$ is Q-linear, i.e., there exists a vector norm $\|\cdot\|$ such that

$$
\limsup _{k \rightarrow \infty} \frac{\left\|x^{(k+1)}-u\right\|}{\left\|x^{(k)}-u\right\|}<1 .
$$

Theorem 3.2 can be proved in a similar argument as [10, Corollary 5.2]. In the following, we will use an alternative way to prove Theorem 3.2. To do this, we need the following two lemmas.

Lemma 3.1 Let $\mathscr{A}$ and $\mathscr{B}$ be as in Theorem 3.1. For any $x \in \operatorname{int}\left(P_{n}\right), F^{\prime}(x)$, the Jacobian of $F$ at $x$, is a primitive matrix.

Proof Let $x \in \operatorname{int}\left(P_{n}\right)$ and $M=F^{\prime}(x)$. Clearly, for any $i, j=1,2, \ldots, n$, $M_{i j}>0$ if and only if $b_{i i_{2} \cdots i_{m}} \neq 0$ for some $i_{l}=j, l=2,3, \ldots, m$. Hence, $\mathscr{G}(M)=\mathscr{G}(\mathscr{B})$. By Theorem 2.4, $\mathscr{B}$ is primitive, so it is irreducible. Therefore, by Definition 2.3 and Proposition $2.2, \mathscr{G}(\mathscr{B})(=\mathscr{G}(M))$ is strongly connected. Therefore, $M$ is irreducible. Since $b_{i} i \cdots i \neq 0$ for $i=1,2, \ldots, n$, we have $M_{i i} \neq 0$, $i=1,2, \ldots, n$. By $[19$, Corollary 3.2], $M$ is primitive.

Lemma 3.2 Let $\mathscr{A}, \mathscr{B}, \lambda$, and $u$ be as in Theorem 3.1, and let $H^{\prime}(u)$ be the Jacobian of the function $H$ at $u$. Then,

$$
\rho\left(H^{\prime}(u)\right)<1 .
$$

Proof For $\lambda$ and $u$ in Theorem 3.1, we have

$$
F(u)=\lambda u^{[m-1]}, \quad \phi(u)=1 .
$$

Hence,

$$
G(u)=(F(u))^{\left[\frac{1}{m-1}\right]}=\lambda^{\frac{1}{m-1}} u .
$$

Let

$$
\lambda_{1}=\lambda^{\frac{1}{m-1}}, \quad V=F^{\prime}(u),
$$

the Jacobian of the function $F$ at $u$. By simple computation, the Jacobian of $G$ at $u$ is

$$
G^{\prime}(u)=\operatorname{diag}\left(\frac{1}{m-1}(F(u))^{\left[\frac{2-m}{m-1}\right]}\right) F^{\prime}(u)=\frac{1}{m-1} \operatorname{diag}\left(\left(\lambda_{1} u\right)^{[2-m]}\right) V .
$$

Since $u$ is a positive vector, we have

$$
\mathscr{G}\left(G^{\prime}(u)\right)=\mathscr{G}(V) .
$$

Hence, by Lemma 3.1, $G^{\prime}(u)$ is a primitive matrix.

Since $G^{\prime}(u)$ is primitive, by Theorem 2.1 , the eigenvalues $v_{1}, v_{2}, \ldots, v_{n}$ of $G^{\prime}(u)$ can be ordered in such a way that

$$
v_{1}=\rho\left(G^{\prime}(u)\right)>\left|v_{2}\right| \geqslant\left|v_{3}\right| \geqslant \cdots \geqslant\left|v_{n}\right|
$$


For all $t>1$, we have

$$
\begin{aligned}
t \lambda_{1} u & =G(t u) \\
& =G(u)+(t-1) G^{\prime}(u) u+o(t-1) \\
& =\lambda_{1} u+(t-1) G^{\prime}(u) u+o(t-1) .
\end{aligned}
$$

Hence,

$$
(t-1) \lambda_{1} u=(t-1) G^{\prime}(u) u+o(t-1),
$$

which implies

$$
G^{\prime}(u) u=\lambda_{1} u .
$$

By Theorem 2.1, $\lambda_{1}=v_{1}$ and $u$ is an eigenvector of $G^{\prime}(u)$ associated with the eigenvalue $v_{1}$.

Since

$$
\phi(G(u))=\phi\left(\lambda_{1} u\right)=\lambda_{1},
$$

by simple computation, we have

$$
H^{\prime}(u)=\frac{G^{\prime}(u)}{\lambda_{1}}-\frac{G(u) e G^{\prime}(u)}{\lambda_{1}^{2}}=\frac{G^{\prime}(u)-u e G^{\prime}(u)}{\lambda_{1}},
$$

where $e$ is the $n$-dimensional row vector of all ones. Let

$$
M=G^{\prime}(u), \quad Q=M-u e M .
$$

In the following, we will prove that the spectral radius of $Q$ is equal to $\left|v_{2}\right|$. To this end, we only need to show that the spectrum of $Q$ is

$$
\sigma(Q)=\left\{0, v_{2}, v_{3}, \ldots, v_{n}\right\}
$$

Since

$$
\phi(u)=e u=1
$$

we have

$$
Q^{\mathrm{T}} e^{\mathrm{T}}=(M-u e M)^{\mathrm{T}} e^{\mathrm{T}}=M^{\mathrm{T}} e^{\mathrm{T}}-M^{\mathrm{T}} e^{\mathrm{T}} u^{\mathrm{T}} e^{\mathrm{T}}=M^{\mathrm{T}} e^{\mathrm{T}}-M^{\mathrm{T}} e^{\mathrm{T}}=0 .
$$

Hence, $e^{\mathrm{T}}$ is an eigenvector of $Q^{\mathrm{T}}$ associated with the eigenvalue 0 . We consider two cases for $M^{\mathrm{T}}$.

Case $1 M^{\mathrm{T}}=G^{\prime}(u)^{\mathrm{T}}$ is diagonalizable, i.e., $M^{\mathrm{T}}$ is semi-simple.

We may assume that for $i=2,3, \ldots, n, w^{i}$ is an eigenvector of $M^{\mathrm{T}}$ associated with the eigenvalue $v_{i}$, and the set $\left\{w^{i}: i=2, \ldots, n\right\}$ is linearly independent. For $i=2,3, \ldots, n$, since

$$
v_{i} u^{\mathrm{T}} w^{i}=u^{\mathrm{T}} M^{\mathrm{T}} w^{i}=\lambda_{1} u^{\mathrm{T}} w^{i},
$$

we obtain $u^{\mathrm{T}} w^{i}=0$. Hence,

$$
Q^{\mathrm{T}} w^{i}=(M-u e M)^{\mathrm{T}} w^{i}=M^{\mathrm{T}} w^{i}-M^{\mathrm{T}} e^{\mathrm{T}} u^{\mathrm{T}} w^{i}=M^{\mathrm{T}} w^{i}=v_{i} w^{i} .
$$


This means that $w^{i}$ is an eigenvector of $Q^{\mathrm{T}}$ associated with the eigenvalue $v_{i}, i=$ $2,3, \ldots, n$. Now, we prove the set $\left\{e^{\mathrm{T}}, w^{i}, i=2, \ldots, n\right\}$ is linearly independent. Suppose

$$
\alpha_{1} e^{\mathrm{T}}+\alpha_{2} w^{2}+\cdots+\alpha_{n} w^{n}=0
$$

and

$$
v_{i} \begin{cases}\neq 0, & i=2,3, \ldots, p \\ =0, & i=p+1, \ldots, n\end{cases}
$$

Then, we have

$$
\alpha_{1} Q^{\mathrm{T}} e^{\mathrm{T}}+\alpha_{2} Q^{\mathrm{T}} w^{2}+\cdots+\alpha_{n} Q^{\mathrm{T}} w^{n}=\alpha_{2} v_{2} w^{2}+\cdots+\alpha_{p} v_{p} w^{p}=0 .
$$

Since set $\left\{w^{i}: i=2, \ldots, n\right\}$ is linearly independent, we obtain

$$
\alpha_{2}=\alpha_{3}=\cdots=\alpha_{p}=0 .
$$

Hence, by (3.4), we have

$$
\alpha_{1} e^{\mathrm{T}}+\alpha_{p+1} w^{p+1}+\cdots+\alpha_{n} w^{n}=0 .
$$

Then,

$$
\begin{aligned}
& \alpha_{1} M^{\mathrm{T}} e^{\mathrm{T}}+\alpha_{p+1} M^{\mathrm{T}} w^{p+1}+\cdots+\alpha_{n} M^{\mathrm{T}} w^{n} \\
& \quad=\alpha_{1} M^{\mathrm{T}} e^{\mathrm{T}}+\alpha_{p+1} v_{p+1} w^{p+1}+\cdots+\alpha_{n} v_{n} w^{n} \\
& \quad=\alpha_{1} M^{\mathrm{T}} e^{\mathrm{T}} \\
& \quad=0 .
\end{aligned}
$$

It follows from $M^{\mathrm{T}} e^{\mathrm{T}}>0$ that $\alpha_{1}=0$. Hence, by (3.5), we have

$$
\alpha_{p+1} w^{p+1}+\cdots+\alpha_{n} w^{n}=0 .
$$

Since set $\left\{w^{i}: i=p+1, \ldots, n\right\}$ is linearly independent, we obtain

$$
\alpha_{p+1}=\cdots=\alpha_{n}=0 .
$$

Therefore,

$$
\alpha_{1}=\cdots=\alpha_{n}=0,
$$

which implies that the set $\left\{e^{\mathrm{T}}, w^{i}, i=2, \ldots, n\right\}$ is linearly independent. Thus, the spectrum of $Q$ is

$$
\sigma(Q)=\sigma\left(Q^{\mathrm{T}}\right)=\left\{0, v_{2}, v_{3}, \ldots, v_{n}\right\}
$$

Case $2 M^{\mathrm{T}}$ is defective.

In this case, we may assume that $M^{\mathrm{T}}$ has distinct eigenvalues $v_{1}\left(=\lambda_{1}\right), v_{2}$, $\ldots, v_{q}, q<n$, and these eigenvalues can be ordered in such a way that

$$
v_{1}\left(=\lambda_{1}\right)>\left|v_{2}\right| \geqslant\left|v_{3}\right| \geqslant \cdots \geqslant\left|v_{q}\right| .
$$


Then, $M^{\mathrm{T}}$ has the form

$$
M^{\mathrm{T}}=X J X^{-1}
$$

where

$$
J=\operatorname{diag}\left\{J_{1}, J_{2}, \ldots, J_{q}\right\}
$$

is in Jordan canonical form (see, e.g., [12]). The Jordan blocks $J_{i}, i=1,2, . ., q$, are square matrices of various sizes, each having the form

$$
J_{i}=\left[\begin{array}{ccccc}
v_{i} & 1 & & & \\
& v_{i} & 1 & & \\
& & \ddots & \ddots & \\
& & & \ddots & 1 \\
& & & & v_{i}
\end{array}\right],
$$

where $v_{i}$ is an eigenvalue of $M^{\mathrm{T}}$. We suppose that $J_{1}=\left[\lambda_{1}\right]$ and $X_{i}$ is the $i$-th column vector of $X, i=1,2, \ldots, n$. For each Jordan block $J_{i}, i=2,3, \ldots, q$, we assume that the size of $J_{i}$ is $l_{i}$. Since $M^{\mathrm{T}}=X J X^{-1}$, we have

$$
\begin{gathered}
M^{\mathrm{T}} X_{2}=v_{2} X_{2}, \\
M^{\mathrm{T}} X_{3}=v_{2} X_{3}+X_{2}, \\
M^{\mathrm{T}} X_{4}=v_{2} X_{4}+X_{3}, \\
\cdots, \\
M^{\mathrm{T}} X_{l_{2}+1}=v_{2} X_{l_{2}+1}+X_{l_{2}}, \\
M^{\mathrm{T}} X_{l_{2}+2}=v_{3} X_{l_{2}+2}, \\
M^{\mathrm{T}} X_{l_{2}+3}=v_{3} X_{l_{2}+2}+X_{l_{2}+1},
\end{gathered}
$$

Similarly, as in Case 1, we have

$$
u^{\mathrm{T}} X_{2}=0, \quad Q^{\mathrm{T}} X_{2}=v_{2} X_{2} .
$$

Then,

$$
v_{2} u^{\mathrm{T}} X_{3}=u^{\mathrm{T}} M^{\mathrm{T}} X_{3}-u^{\mathrm{T}} X_{2}=\lambda_{1} u^{\mathrm{T}} X_{3},
$$

and we obtain $u^{\mathrm{T}} X_{3}=0$. Hence,

$$
Q^{\mathrm{T}} X_{3}=(M-u e M)^{\mathrm{T}} X_{3}=M^{\mathrm{T}} X_{3}-M^{\mathrm{T}} e^{\mathrm{T}} u^{\mathrm{T}} X_{3}=v_{2} X_{3}+X_{2} .
$$

Similarly, we have

$$
\begin{gathered}
Q^{\mathrm{T}} X_{4}=v_{2} X_{4}+X_{3}, \\
\cdots, \\
Q^{\mathrm{T}} X_{l_{2}+1}=v_{2} X_{l_{2}+1}+X_{l_{2}}, \\
Q^{\mathrm{T}} X_{l_{2}+2}=v_{3} X_{l_{2}+2}, \\
Q^{\mathrm{T}} X_{l_{2}+3}=v_{3} X_{l_{2}+2}+X_{l_{2}+1},
\end{gathered}
$$


Similarly, as in Case 1 , we can prove the set $\left\{e^{\mathrm{T}}, X_{i}, i=2, \ldots, n\right\}$ is linearly independent. Let $Y=\left[e^{\mathrm{T}}, X_{i}, i=2, \ldots, n\right]$. Then we have

$$
Q^{\mathrm{T}} Y=Y \operatorname{diag}\left\{[0], J_{2}, \ldots, J_{q}\right\}
$$

Therefore, the spectrum of $Q$ is

$$
\sigma(Q)=\sigma\left(Q^{\mathrm{T}}\right)=\left\{0, v_{2}, v_{3}, \ldots, v_{q}\right\} .
$$

In summary, we have the spectral radius of $Q$,

$$
\rho(Q)=\left|v_{2}\right|\left(<\lambda_{1}\right)
$$

Therefore,

$$
\rho\left(H^{\prime}(u)\right)=\rho\left(\frac{Q}{\lambda_{1}}\right)=\frac{\left|v_{2}\right|}{\lambda_{1}}<1 .
$$

Now, we give the proof of Theorem 3.2 as follows.

Proof of Theorem 3.2 From Proposition 2.1 and Lemma 3.2, there exist an $\varepsilon>0$ and a spectral norm $\|\cdot\|_{\varepsilon} \in \mathscr{N}$ such that

$$
\left\|H^{\prime}(u)\right\|_{\varepsilon} \leqslant \rho\left(H^{\prime}(u)\right)+\varepsilon<1 .
$$

It follows from (3.3) that

$$
x^{(k+1)}-u=H\left(x^{(k)}\right)-H(u)=H^{\prime}(u)\left(x^{(k)}-u\right)+o\left(\left\|x^{(k)}-u\right\|_{\varepsilon}\right) .
$$

Since $\left\|x^{(k)}-u\right\|_{\varepsilon}$ tends to 0 as $k$ goes to infinity, we obtain

$$
\limsup _{k \rightarrow \infty} \frac{\left\|x^{(k+1)}-u\right\|_{\varepsilon}}{\left\|x^{(k)}-u\right\|_{\varepsilon}} \leqslant\left\|H^{\prime}(u)\right\|_{\varepsilon} \leqslant \rho\left(H^{\prime}(u)\right)+\varepsilon<1,
$$

which means that the convergence rate of the sequence $\left\{x^{(k)}\right\}$ is linear.

In Theorem 3.2, we have established the Q-linear convergence of Algorithm 3.1 for weakly irreducible nonnegative tensors. Numerical results reported in $[18,20,32]$ show that Algorithm 3.1 performs well for irreducible nonnegative tensors. However, for some reducible nonnegative tensors, Algorithm 3.1 may not produce the largest eigenvalue. Consider the following three examples.

Example 3.1 The order-3 3-dimensional tensor $\mathscr{D}$ is given by

$$
\begin{gathered}
d_{111}=d_{333}=1, \quad d_{222}=2, \\
d_{i j k}=0, \quad \text { elsewhere. }
\end{gathered}
$$

Example 3.2 The order-3 3-dimensional tensor $\mathscr{F}$ given by

$$
\begin{gathered}
f_{111}=f_{333}=f_{121}=f_{231}=1, \quad f_{222}=2, \\
f_{i j k}=0, \quad \text { elsewhere. }
\end{gathered}
$$


Example 3.3 The order-3 3-dimensional tensor $\mathscr{M}$ given by

$$
\begin{aligned}
m_{111}= & m_{333}=m_{121}=1, \quad m_{222}=2, \\
& m_{i j k}=0, \quad \text { elsewhere }
\end{aligned}
$$

By Proposition $2.2, \mathscr{D}, \mathscr{F}$, and $\mathscr{M}$ are reducible tensors because $\mathscr{G}(\mathscr{D})$, $\mathscr{G}(\mathscr{F})$, and $\mathscr{G}(\mathscr{M})$ are not strongly connected, respectively. We choose $x^{(1)}=$ $[10,10,10]^{\mathrm{T}}$. By Algorithm 3.1, we cannot obtain the largest eigenvalue for these tensors within 1000 iterations.

For a nonnegative tensor, a weak Perron-Frobenius theorem has been given in [30], which we state as follows.

Theorem 3.3 [30] If $\mathscr{A}$ is a nonnegative tensor of order $m$ and dimension $n$, then $\rho(\mathscr{A})$ is an eigenvalue of $\mathscr{A}$ associated with a nonnegative eigenvector $y \in \mathbb{R}^{n}, y \neq 0$.

\section{An algorithm for spectral radius of any nonnegative tensors}

In this section, based on Theorem 3.3, we present a convergent algorithm for computing the spectral radius for any nonnegative tensor $\mathscr{A}$.

Let $\mathscr{E}$ denote the order- $m n$-dimensional tensor with every entry being one. For any nonnegative tensor $\mathscr{A}$ and any $\tau>0, \mathscr{A}+\tau \mathscr{E}$ is an irreducible nonnegative tensor.

Algorithm 4.1 Let $t o l>0$ be given. Choose a sequence

$$
\tau_{1}>\tau_{2}>\cdots>\tau_{k}>\cdots>0
$$

satisfying $\lim _{k \rightarrow \infty} \tau_{k}=0$. Compute the largest eigenvalue $\lambda$ of $\mathscr{A}$ and corresponding eigenvector $u$ by using Algorithm 3.1. If Algorithm 3.1 can produce $\lambda$ and $u$ successfully, then stop. Otherwise, go to next step.

For $k=1,2, \ldots$, let $\mathscr{A}^{k}=\mathscr{A}+\tau_{k} \mathscr{E}$ and do

1. Compute the largest eigenvalue $\lambda^{k}$ of $\mathscr{A}^{k}$ and corresponding eigenvector $u^{k}$ by using Algorithm 3.1.

2. If $k \geqslant 2$ and $\left\|\lambda^{k}-\lambda^{k-1}\right\| \leqslant$ tol then output $u^{k}$ and $\lambda^{k}$, and terminate the algorithm.

\section{End}

Theorem 4.1 Suppose that $\mathscr{A}$ is a nonnegative tensor and $\rho(\mathscr{A})$ is the spectral radius of $\mathscr{A}$. Then, Algorithm 4.1 produces the spectral radius $\rho(\mathscr{A})$ and a corresponding eigenvector $u$, or generates two convergent sequences $\left\{\lambda^{k}\right\}$ and $\left\{u^{k}\right\}$ such that

$$
\lim _{k \rightarrow \infty} \lambda^{k}=\rho(\mathscr{A}), \quad \lim _{k \rightarrow \infty} u^{k}=u
$$

respectively. 
Proof See the proof of [30, Theorem 2.3].

In order to show the viability of Algorithm 4.1, we used Matlab to test it on Examples 3.1-3.3. Throughout the computational experiments, we choose

$$
\text { tol }=10^{-6}, \quad \tau_{k}=0.001 \times 10^{-k}, \quad k=1,2, \ldots
$$

Our numerical results are reported in Table 1, which show that Algorithm 4.1 is able to produce the largest eigenvalue for reducible nonnegative tensors. In Table $1, k$ denotes the number of iterations, $\lambda^{k}$ and $u^{k}$ denote the largest eigenvalue of $\mathscr{A}^{k}$ and corresponding eigenvector at the final iteration, respectively.

Table 1 Numerical results of Algorithm 4.1 for Examples 3.1-3.3

\begin{tabular}{crcc}
\hline Example & $k$ & $\lambda^{k}$ & $u^{k}$ \\
\hline 3.1 & 4 & 2.0000 & $(0.0003,1.0000,0.0003)^{\mathrm{T}}$ \\
3.2 & 11 & 2.0000 & $(0.7071,0.7071,0.0000)^{\mathrm{T}}$ \\
3.3 & 5 & 2.0000 & $(0.7071,0.7071,0.0001)^{\mathrm{T}}$ \\
\hline
\end{tabular}

To conclude this section and the whole paper, we remark that ill-poses problem may happen when the parameter $\tau_{k}$ in Algorithm 4.1 is small enough. Thus, we need a stable procedure to control the sequence $\left\{\tau_{k}\right\}$, which is one of our future research topics.

Acknowledgements Zhou's work was supported by the Australian Research Council. Qi's work was supported by the Hong Kong Research Grant Council (Grant Nos. PolyU 501808, 501909, 502510 502111).

\section{References}

1. Bloy L, Verma R. On computing the underlying fiber directions from the diffusion orientation distribution function. In: Medical Image Computing and ComputerAssisted Intervention MICCAI 2008. Berlin/Heidelberg: Springer, 2008, 1-8

2. Bulò S R, Pelillo M. New bounds on the clique number of graphs based on spectral hypergraph theory. In: Stützle T, ed. Learning and Intelligent Optimization. Berlin: Springer-Verlag, 2009, 45-48

3. Chang K C, Pearson K, Zhang T. Perron-Frobenius theorem for nonnegative tensors. Commun Math Sci, 2008, 6: 507-520

4. Chang K C, Pearson K, Zhang T. On eigenvalue problems of real symmetric tensors. J Math Anal Appl, 2009, 350: 416-422

5. Chang K C, Pearson K, Zhang T. Primitivity, the convergence of the NQZ method, and the largest eigenvalue for nonnegative tensors. SIAM J Matrix Anal Appl, 2011, 32: 806-819

6. Chang K C, Qi L, Zhou G. Singular values of a real rectangular tensor. J Math Anal Appl, 2010, 370: 284-294

7. Collatz L. Einschliessungssatz für die charakteristischen Zahlen von Matrizen. Math Z, 1942, 48: 221-226

8. De Lathauwer L, De Moor B, Vandewalle J. On the best rank-1 and rank- $\left(R_{1}, \ldots, R_{N}\right)$ approximation of higher-order tensors. SIAM J Matrix Anal Appl, 2000, 21: 1324-1342 
9. Drineas P, Lim L-H. A multilinear spectral theory of hyper-graphs and expander hypergraphs. 2005

10. Friedland S, Gaubert S, Han L. Perron-Frobenius theorem for nonnegative multilinear forms and extensions. Linear Algebra Appl, 2013, 438: 738-749

11. Golub G H, Loan C F V. Matrix Computations. Baltimore: Johns Hopkins University Press, 1996

12. Horn R A, Johnson C R. Matrix Analysis. Cambridge: Cambridge University Press, 1985

13. Hu S, Huang Z, Qi L. Finding the spectral radius of a nonnegative tensor. Department of Applied Mathematics, The Hong Kong Polytechnic University, 2010

14. Kofidis E, Regalia P A. On the best rank-1 approximation of higher-order supersymmetric tensors. SIAM J Matrix Anal Appl, 2002, 23: 863-884

15. Kolda T G, Bader B W. Tensor decompositions and applications. SIAM Rev, 2009, 51 $455-500$

16. Lim L -H. Singular values and eigenvalues of tensors: a variational approach. In: Proceedings IEEE International Workshop on Computational Advances in Multi-Sensor Adaptive Processing (CAMSAP '05), Vol 1. 2005, 129-132

17. Lim L-H. Multilinear pagerank: measuring higher order connectivity in linked objects. In: The Internet: Today and Tomorrow. July, 2005

18. Liu Y, Zhou G, N. F. Ibrahim N F. An always convergent algorithm for the largest eigenvalue of an irreducible nonnegative tensor. J Comput Appl Math, 2010, 235: 286292

19. Minc H. Nonnegative Matrices. New York: John Wiley and Sons, Inc, 1988

20. Ng M, Qi L, Zhou G. Finding the largest eigenvalue of a non-negative tensor. SIAM J Matrix Anal Appl, 2009, 31: 1090-1099

21. Ni Q, Qi L, Wang F. An eigenvalue method for testing the positive definiteness of a multivariate form. IEEE Trans Automatic Control, 2008, 53: 1096-1107

22. Pearson K J. Essentially positive tensors. Int J Algebra, 2010, 4: 421-427

23. Qi L. Eigenvalues of a real supersymmetric tensor. J Symbolic Comput, 2005, 40: 1302-1324

24. Qi L. The spectral theory of tensors. Department of Applied Mathematics, The Hong Kong Polytechnic University, 2012

25. Qi L, Wang F, Wang Y. Z-eigenvalue methods for a global polynomial optimization problem. Math Program, 2009, 118: 301-316

26. Qi L, Wang Y, Wu E X. D-eigenvalues of diffusion kurtosis tensor. J Comput Appl Math, 2008, 221: 150-157

27. Shashua A, Hazan T. Non-negative tensor factorization with applications to statistics and computer vision. In: International Conference of Machine Learning (ICML), August, 2005

28. Varga R. Matrix Iterative Analysis. Englewood Cliffs: Prentice-Hall, Inc, 1962

29. Wood R J, O'Neill M J. Finding the spectral radius of a large sparse non-negative matrix. ANZIAM J, 2007, 48: C330-C345

30. Yang Y, Yang Q. Further results for Perron-Frobenius theorem for nonnegative tensors. SIAM J Matrix Anal Appl, 2010, 31: 2517-2530

31. Zhang L, Qi L. Linear convergence of an algorithm for computing the largest eigenvalue of a nonnegative tensor. Numer Linear Algebra Appl, 2012, 19: 830-841

32. Zhang L, Qi L, Xu Y. Finding the largest eigenvalue of an irreducible nonnegative tensor and linear convergence for weakly positive tensors. J Comput Math, 2012, 30: $24-33$ 